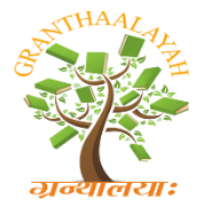

\author{
INTERNATIONAL JOURNAL OF RESEARCH - \\ GRANTHAALAYAH \\ A knowledge Repository
}

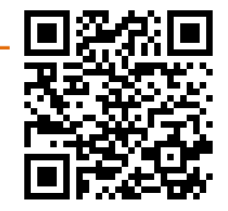

Science

\title{
CASE REPORT - ACUPUNCTURE TREATMENT IN MALE PATIENT WITH HASHIMOTO THYROIDITIS
}

\author{
Blagica Arsovska ${ }^{1,2}$, Jihe Zhu ${ }^{1}$, Kristina Kozovska 1,3 \\ ${ }^{1}$ Faculty of Medical Sciences, University Goce Delchev, Shtip, Republic of Macedonia \\ ${ }^{2}$ Institute of Biology, Faculty of Natural Sciences and Mathematics, Skopje, Republic of \\ Macedonia \\ ${ }^{3}$ Medicine Faculty, St. Cyril and Methodius University of Skopje, Republic of Macedonia
}

\begin{abstract}
Hashimoto's thyroiditis is associated with the presence of anti-thyroid autoantibodies (anti-TPO). High serum antibodies are found in the active phases of chronic autoimmune thyroiditis. In many medical cases with Hashimoto's thyroiditis it is possible to have high levels of anti-TPO antibodies and fT4, fT3 and TSH levels to be within the normal range. Most doctors believe that Hashimoto's thyroiditis is an incurable form of thyroid deficiency. With the TCM treatment the patient's strength and vitality can be improved, the Qi flow can be harmonized, nourished and strengthened and the body's system can be repaired and recharged so the patient may go into remission. The treated patient is 44 year old man, diagnosed with Hashimoto's thyroiditis caused by stress. The patient has done 40 acupuncture treatments within 21 months, once a week. The patient wasn't taking any additional hormonal medications. The parameters for fT4, fT3 and TSH were showing normal levels and the anti-TPO levels were increased. Before the treatment the anti-TPO antibodies amount was $252.4 \mathrm{IU} / \mathrm{ml}$ and after the treatment $4.07 \mathrm{IU} / \mathrm{ml}$ (normal $<80 \mathrm{IU} / \mathrm{ml}$ ). Treated points are: BL15 (XinShu), BL20 (PiShu), BL23 (ShenShu), DU4 (MingMen), DU14 (DaZhui), DU20 (BaiHui), ST9 (RenYing), LI4 (HeGu), KI3 (TaiXi), GB20 (FengChi), RN4 (QuanYuan), RN6 (QiHai), LR2 (TaiChong), SP9 (YinLingQuan), SP6 (SanYinJiao), ST36 (ZuSanLi) and Ashi points located on the neck (front and back).
\end{abstract}

Keywords: Traditional Chinese Medicine; Acupuncture; Thyroid; Hashimoto.

Cite This Article: Blagica Arsovska, Jihe Zhu, and Kristina Kozovska. (2019). "CASE REPORT - ACUPUNCTURE TREATMENT IN MALE PATIENT WITH HASHIMOTO THYROIDITIS.” International Journal of Research - Granthaalayah, 7(9), 288-291. https://doi.org/10.29121/granthaalayah.v7.i9.2019.612.

\section{Introduction}

Hashimoto's thyroiditis is associated with the presence of anti-thyroid autoantibodies (anti-TPO). High serum antibodies are found in the active phases of chronic autoimmune thyroiditis. In many 
medical cases with Hashimoto's thyroiditis it is possible to have high levels of anti-TPO antibodies and $\mathrm{fT} 4$, fT3 and TSH levels to be within the normal range.

Hashimoto's thyroiditis is one of the most frequently seen forms of hypothyroidism and can be a very complex and chronic long-term autoimmune disease. [1] Most doctors believe that Hashimoto's thyroiditis is an incurable form of thyroid deficiency.

Symptoms that may be present are: fatigue, weight gain, feeling cold, excessive sleepiness, constipation, depression, dry skin and hair, infertility and etc.

Due to our modern lifestyle and stress, the incidence of the disease is increasing and when treating Hashimoto two causes must be taken into account: emotional damage and over-ambitious tendencies. The factors for occurrence for this causes are lack of rest and too much drive. The negative emotions as anger, fear, depression, excessive anxiety and excessive thoughts can disrupt the function of the liver Qi flow and Qi stagnation may occur. Qi stagnation can cause accumulation of body fluids which manifest as phlegm and affects the thyroid gland. In terms of Traditional Chinese Medicine (TCM) in most cases the problem comes from reduced energy in the Spleen and Kidney. The Kidney organ in TCM is connected to the hair, thus the Kidney Yang and Essence are reduced the hair becomes thin, fragile and dull and starts to fall off. [2]

TCM is not treating only the symptoms, but the root of the disease and has a very holistic approach on the problem. In TCM every person is treated individually and all aspects are taken into consideration - mental, emotional, physical, social and spiritual. With the acupuncture and Chinese herbs as part of the TCM treatment, it can be helped in the management of the condition.

With the TCM treatment the patient's strength and vitality can be improved, the Qi flow can be harmonized, nourished and strengthened and the body's system can be repaired and recharged so the patient may go into remission. [1]

\section{Case Report}

The treated patient is 44 year old man, diagnosed with Hashimoto's thyroiditis caused by stress. The patient was feeling tiredness, loss of energy, emotional stress and intense hair loss. The patient has done 40 acupuncture treatments within 21 months, once a week. The patient wasn't taking any additional hormonal medications. Treatments are made in a clinic for Traditional Chinese Medicine and acupuncture in Skopje, North Macedonia, by a doctor specialist in acupuncture. Treatments were made indoor, on a room temperature, with duration of 30-45 minutes. In the treatment were used fine sterile disposable needles with dimensions $0.25 \times 25 \mathrm{~mm}$ manufactured by Wuijuiang City Medical \& Health Material Co., LTD.

The parameters for fT4, fT3 and TSH were showing normal levels and the anti-TPO levels were increased. Before the treatment the anti-TPO antibodies amount was $252.4 \mathrm{IU} / \mathrm{ml}$ and after the treatment $4.07 \mathrm{IU} / \mathrm{ml}$ (normal $<80 \mathrm{IU} / \mathrm{ml}$ ). After the treatment the patient grew new hair. The results from the blood test are shown on table 1. 
Table 1: Blood test results

\begin{tabular}{|l|l|l|l|}
\hline Date & Anti-TPO $(<\mathbf{8 0} \mathbf{~ I U} / \mathbf{m l})$ & TSH $(\mathbf{0 . 4 5 - 4 . 6 7} \mathbf{~ m I U} / \mathbf{m l})$ & $\mathbf{f T 4}(\mathbf{0 . 7 1 - 1 . 8 5} \mathbf{~ n g} / \mathbf{d l})$ \\
\hline 01.09 .17 & 252.4 & 1.41 & 0.93 \\
\hline 27.03 .18 & 112 & 1.965 & 0.89 \\
\hline 17.10 .18 & 144.8 & 1.21 & 1.20 \\
\hline 12.06 .19 & 40.07 & $/$ & $/$ \\
\hline
\end{tabular}

Treated points are: BL15 (XinShu), BL20 (PiShu), BL23 (ShenShu), DU4 (MingMen), DU14 (DaZhui), DU20 (BaiHui), ST9 (RenYing), LI4 (HeGu), KI3 (TaiXi), GB20 (FengChi), RN4 (QuanYuan), RN6 (QiHai), LR2 (TaiChong), SP9 (YinLingQuan), SP6 (SanYinJiao), ST36 ( $\mathrm{ZuSanLi}$ ) and Ashi points located on the neck (front and back).

When the anti-TPO antibodies are elevated it represents that inflammatory event occurred in the thyroid gland. The thyroid may not be completely destroyed and still can be able to release iodothyronines in sufficient amount, but in time overt hypothyroidism may occur. 80-90\% of the people with Hashimoto's thyroiditis have increased either anti-TPO or anti-TG antibodies, or both. If the thyroid antibodies are more elevated there is a greater chance of developing overt hypothyroidism and additional autoimmune conditions. It is also possible a patient to have Hashimoto's thyroiditis and negative test for thyroid antibodies. This form is called serum negative Hashimoto's thyroiditis and is a less aggressive. Thyroid antibodies under $100 \mathrm{IU} / \mathrm{mL}$ are labeled as "remission" status, and thyroid antibodies above $500 \mathrm{IU} / \mathrm{mL}$ are labeled as "aggressive". A complete remission is possible, when the patient is no longer testing positive for any thyroid antibodies, doesn't have a single symptom and there are no evidences of damage to the thyroid gland. [3]

In the treated case it was achieved complete remission with acupuncture treatment.

Acupuncture can regulate the emotional state of the patient, the hormones, the autonomic nervous system, regulate the flow the Qi energy, improve sleeping, reduce stress and etc. [4] [5]

\section{Conclusion}

Acupuncture as part of the TCM can successfully treat Hashimoto's thyroiditis and achieve complete remission and optimal quality of life in the treated patients.

\section{References}

[1] Ling S; How to Treat Hashimoto's Thyroiditis With Chinese Medicine!; 2017 [www.sustainhealth.com.au]

[2] Malikov D; Traditional Chinese Medicine Approach to Hypothyroidism; Int J Complement Alt Med 5(1): 00142. DOI: 10.15406/ijcam.2017.05.00142

[3] Wentz I; Thyroid Antibodies Part 1: An Early Warning for Thyroid Disease; 2018 [www.thyroidpharmacist.com]

[4] Luzina C, Xin N; Autoimmune thyroiditis - an ancient approach of traditional Chinese medicine to a modern disease; Archiv Euromedica 1st \& 2nd Edition 2011 
[5] Zhu J, Arsovska B, Kozovska K; Case Report: Acupuncture Treatment in Male Patient With Primary Hypothyroidism; International Journal of Research - Granthaalayah, 7(3), 217-220. https://doi.org/10.5281/zenodo.2631392.

\footnotetext{
*Corresponding author.

E-mail address: tongdatang-tcm@ hotmail.com
} 